\title{
Addendum: Meteoritic evidence for a Ceres-sized water-rich carbonaceous chondrite parent asteroid
}

\author{
V. E. Hamilton (D), C. A. Goodrich, A. H. Treiman (D, H. C. Connolly Jr, M. E. Zolensky and M. H. Shaddad
}

Addendum to Nature Astronomy https://doi.org/10.1038/s41550-020-01274-Z, published online 21 December 2020.

In our original Letter, we presented mineralogical and geochemical evidence for prograde metamorphism on a previously unrecognized, large ( 640-1800 km diameter) carbonaceous chondrite (CC) parent body from Almahata Sitta (AhS) 202 (C-ung), a clast in the AhS polymict meteorite. It has been drawn to our attention that some of the mineralogical textures shown in our figures may be inadequately described for the non-specialist reader, so here we provide a clarifying figure and discussion.

One of the key observations that led us to favour prograde, rather than retrograde, metamorphism is the cross-cutting textural relationships between minerals in AhS 202. Figure 1 shows the mineral diopside occurring as irregular remnants surrounded by euhedral tremolite (amphibole) crystals, indicating the consumption of diopside as the tremolite crystals grow. In both prograde and retrograde reaction scenarios, the diopside predates the formation of tremolite, but in a retrograde reaction, the diopside must be inferred to be a relict primary mineral in chondrules. Such a relict nature is implausible as unaltered chondrules in CCs do not contain chemically pure diopside or even substantial amounts of augite as individual grains. Furthermore, Ca-rich pyroxenes, particularly chemically pure diopside, are not a predicted product of high-temperature anhydrous metamorphism of a primary CC assemblage; instead, the Ca component is in anorthite ${ }^{1}$. In the prograde scenario, the presence of diopside is plausible because chemically pure diopside is a common product of lower greenschist facies metamorphism of mafic and ultramafic rocks ${ }^{1}$. In short, our interpretation of prograde metamorphism is grounded in numerous observations of low-temperature aqueous alteration in CCs and is an extension of such cases to higher water contents.

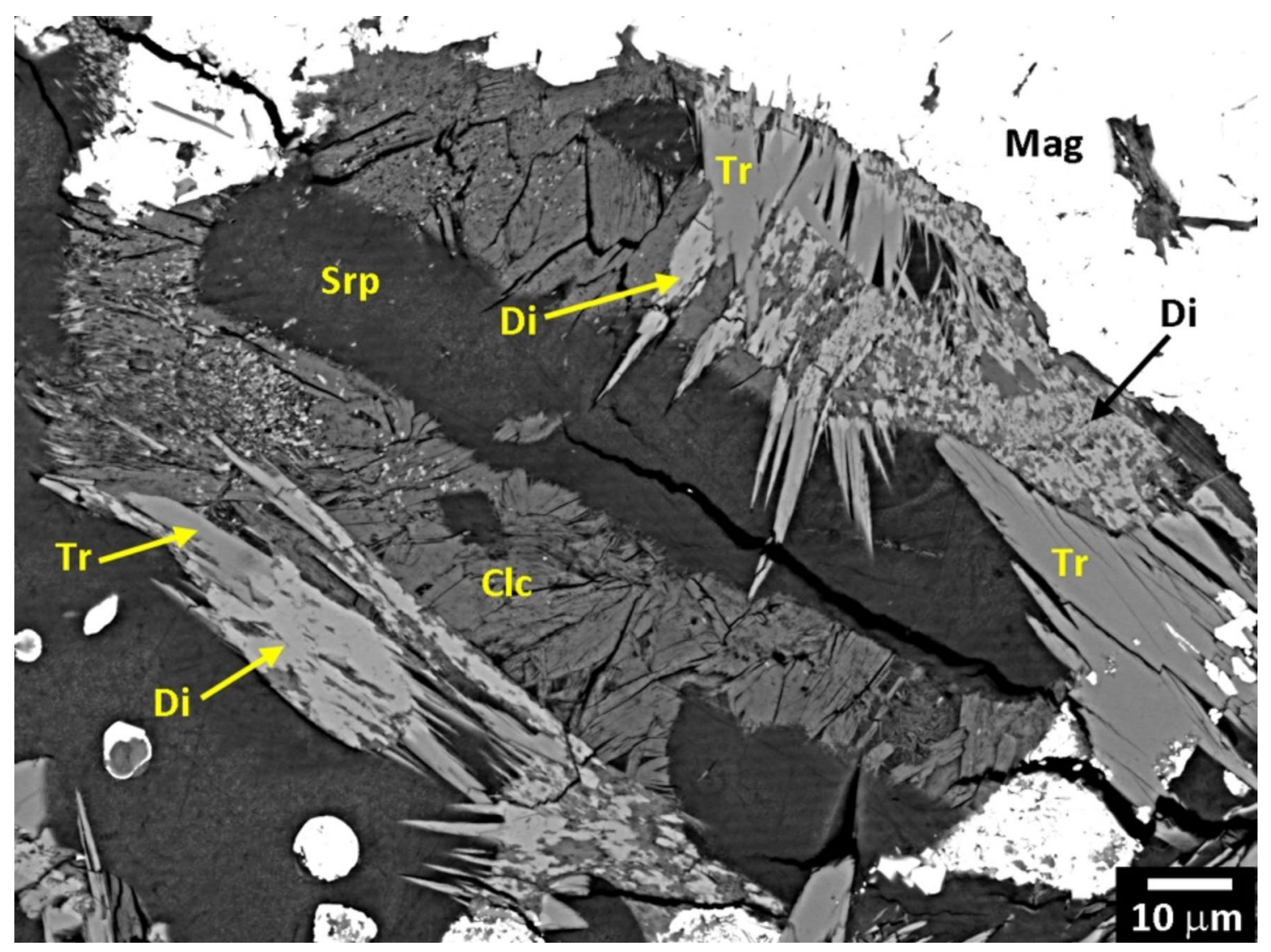

Fig. 1 | Back-scattered electron image of AhS 202. Brighter areas represent higher atomic numbers. Some textural relationships between tremolite (Tr), clinochlore (Clc), diopside ( $\mathrm{Di}$ ), serpentine (Srp), and magnetite (Mag) can be seen. Diopside commonly occurs in patchy/porous textures indicating that it is being resorbed to produce well-formed crystals of tremolite. Serpentine and clinochlore also are cut by tremolite and diopside, further supporting a prograde reaction. 
By comparison, as we discuss in our paper, an occurrence of amphibole in another CC meteorite, Allende (CV3), is attributed to retrograde metamorphism by Brearley ${ }^{2}$, but that example is not directly applicable to our observations for two reasons: first, the Allende amphibole is magnesio-hornblende, which contains $\sim 40$ times more $\mathrm{Al}_{2} \mathrm{O}_{3}$ than the tremolite in $\mathrm{AhS} 202$, thus changing the geochemical reactions that are involved and supporting different metamorphic facies and precursor mineralogy. Second, the Allende mineral textures are clearly retrograde, with thin, TEM-scale, films and veinlets of hydrous silicates cross-cutting a primary chondrule mineral (enstatite), whereas the textures in AhS 202 indicate a hydrous silicate (tremolite) replacing diopside, which, as we explain above, is not a primary mineral in chondrules.

In summary, we reaffirm our interpretation of the origin of abundant tremolite in AhS 202 as resulting from prograde, fluid-assisted, metamorphic reactions at pressures consistent with a larger parent body than has been inferred previously for CCs. The sheer volumetric abundance and composition of amphibole identified in AhS 202 are unique among CCs, which also supports our assertion that whatever conditions resulted in its formation are not well represented in our meteorite collections. As such, AhS 202 illustrates bias in available CCs, as suggested previously ${ }^{3,4}$.

\section{References}

1. Bucher, K. \& Grapes, R. Petrogenesis of Metamorphic Rocks 8th edn (Springer, 2011).

2. Brearley, A. J. Disordered biopyriboles, amphibole, and talc in the Allende meteorite: Products of nebular or parent body aqueous alteration? Science 276, 1103-1105 (1997).

3. Sears, D. W. G. The case for rarity of chondrules and calcium-aluminum-rich inclusions in the early solar system and some implications for astrophysical models. Astrophys. J. 498, 773-778 (1998).

4. Flynn, G. J., Consolmagno, G. J., Brown, P. \& Macke, R. J. Physical properties of the stone meteorites: Implications for the properties of their parent bodies. Chem. Erde 78, 269-298 (2018).

Published online: 25 October 2021

https://doi.org/10.1038/s41550-021-01496-9

(C) Springer Nature Limited 2021 